\title{
A histochemical method of differentiating lower gastrointestinal tract mucin from other mucins in primary or metastatic tumours
}

\author{
C. F. A. CUlling, P. E. REID, J. D. BURTON, AND W. L. DUNN \\ From the Department of Pathology, University of British Columbia and Vancouver General Hospital, \\ Vancouver, British Columbia, Canada
}

SYNOPSIS Epithelial mucins of the normal terminal ileum, caecum, colon, and rectum of man are unique in that they alone exhibit staining following the periodate-borohydride/ $\mathrm{KOH} / \mathrm{PAS}$ technique. Application of this technique enables one to differentiate those mucin-producing metastases arising from adenocarcinoma of the lower gastrointestinal tract from those arising elsewhere, and may occasionally be useful in determining the site of the primary tumour when it is in doubt. Furthermore, it was found to be especially useful in distinguishing between primary adenocarcinoma of the lung and metastases in the lung from adenocarcinoma of the lower gastrointestinal tract.

We have shown that, in certain defined areas of the gastrointestinal tract, there is an increase in periodic acid-Schiff (PAS) reactivity following treatment with potassium hydroxide $(\mathrm{KOH})$, and that this effect is due to the removal of O-acyl esters from the side chains of sialic acids in epithelial mucins (Culling et al, 1971, 1974; Reid et al, 1973). Originally, detection of this increase in PAS reactivity required a comparison microscope but we have shown that periodate oxidation followed by reduction with sodium borohydride abolished the normal PAS reactivity of tissue, and the subsequent application of the KOH/PAS technique ensures that only its effect is seen (Reid et al, 1973).

This paper describes the use of the periodateborohydride $\mathrm{KOH} / \mathrm{PAS}$ technique as a means of distinguishing between mucins secreted by metastases which have arisen from adenocarcinoma of the lower gastrointestinal tract and those mucins which have been secreted by normal or malignant cells from other tissues. This technique should be especially valuable where the metastases have grown more rapidly than the primary lesion, the former being the presenting complaint.

\section{Materials and Methods}

NORMAL HUMAN TISSUES

Routinely formalin fixed, paraffin processed blocks of the following normal tissues were examined: Received for publication 17 March 1975. parotid, submaxillary, and submandibular glands, liver, thyroid, bronchus, lung, eye, seminal vesicle, aorta, testis, prostate, ovary, uterus, cervix, fallopian tube, kidney; and stomach, small intestine, caecum, colon, and rectum. In addition, there were the numerous relatively normal tissues (for example, liver, lung, breast, ovaries, lymph nodes) containing tumours or metastases.

NEOPLASTIC TISSUES

Formalin fixed, paraffin processed blocks from $89 \underline{3}$ primary adenocarcinomas of colon (49), rectum (2), \& caecum (2), stomach (10), small intestine (1), lung (20), breast (3), bile duct (1), and ovary (1) were $ᄋ$ examined together with 177 blocks of their metastases.

MICROSCOPICAL EXAMINATION Specimens were examined without the observer being aware of the site of the primary tumour.

Staining methods (Culling, 1974)

1 Haematoxylin and eosin (H and E) was used to establish the diagnosis and identify tissues.

2 Periodic acid-Schiff reaction (PAS) was used as a 7 control.

3 Periodate borohydride/potassium hydroxide/PAS technique (PB/KOH/PAS) was used to demonstrate the KOH/PAS reaction in isolation.

Paraffin sections of routine formol-calcium or buffered formalin fixed tissues may be used. 


\section{METHOD}

1. Sections were brought to water, and

2. oxidized with $1 \%$ aqueous periodic acid at room temperature for 60 minutes, and

3. washed in water.

4. They were then reduced in sodium borohydride as follows:

\section{Solution $A$}

$2.45 \%$ boric acid in distilled water; this solution may be stored in the refrigerator.

\section{Solution $B$}

$1.89 \mathrm{~g}$ sodium borohydride dissolved in $167 \mathrm{ml}$ of distilled water; this solution must be made immediately before use.

Slides were placed in a glass (or stainless steel) slide holder (of the bottomless variety, not a coplin jar). This was placed in a container which held 300 $\mathrm{ml}$ yet was narrow enough for the sections to be completely covered by the addition of $100 \mathrm{ml}$ of solution $\mathrm{A}$; this container must be kept cool, by surrounding it with crushed ice. Solution B was then added gradually over a period of 30 minutes and the sections were left for an additional hour; this step should be carried out in a fume hood. They were then washed in running water for 10-15 minutes.

5. Sections were treated in $0.5 \%$ potassium hydroxide in $70 \%$ alcohol at room temperature for 30 minutes, 6. washed in $70 \%$ alcohol, then in water; and

7. stained by the periodic acid-Schiff

(PAS) technique,

8. dehydrated, cleared, and mounted in HSR.

\section{RESULTS}

Mucin showing any red coloration is interpreted as being $\mathrm{KOH} / \mathrm{PAS}$ positive, provided the slide treated by the PB/PAS technique (see below) is unstained. Optionally, sections may be lightly counterstained with haematoxylin, or tartrazine in cellosolve, which will visualize the tissue and will aid the orientation of the section; we preferred the former.

\section{RAPID PERIODATE-BOROHYDRIDE/KOH/PAS TECHNIQUE}

This method, which utilizes a modification of Lillie and Pizzalato's borohydride aldehyde blocking method, can be used as a routine method. However, if a particular section treated by the periodate borohydride/PAS technique (see below), which is used as a negative control, shows any red colour, then the long method (above) should be used.

1. Sections were brought to water and then treated with $1 \%$ periodic acid for 30 minutes,
2. washed in running water for 10 minutes, and

3. treated in $0.1 \%$ sodium borohydride in $1 \%$ disodium hydrogen phosphate for $\mathbf{3 0}$ minutes,

4. slides were washed in water.

Steps 5, 6, 7, and 8 and results as in method above.

PERIODATE BOROHYDRIDE/PAS TECHNIQUE (PB/PAS)

This technique was used to ensure that normal PAS reactivity had been abolished. The method consists of steps 1, 2, 3, and 4 (above) followed by the routine PAS technique and should be performed in parallel with the above method (PB/KOH/PAS). Any staining present in this section will indicate that the periodic acid-borohydride treatment is incomplete due to either (1) insufficient time of oxidation with periodic acid; or (2) incomplete reduction by sodium borohydride. The substitution of simple Schiff reagent for 30 minutes in place of a full PAS method will help to determine the cause since a positive result by the latter indicates that the reduction by sodium borohydride is incomplete.

\section{Results}

PATHOLOGICAL TISSUES

These are summarized in the table and it will be seen that, of the 89 tumours and 177 metastases examined, in no instance was a metastasis positive by the $\mathrm{KOH} / \mathrm{PAS}$ technique when the primary tumour was negative. With one single exception, only those tumours that had arisen in the caecum, colon or rectum gave a positive $\mathrm{KOH} / \mathrm{PAS}$ reaction, and on no occasion did a metastasis from a negative tumour give a positive result.

One primary tumour of the stomach was itself $\mathrm{KOH} / \mathrm{PAS}$ positive and gave rise to metastases (3) which were also $\mathrm{KOH} / \mathrm{PAS}$ positive. The primary

\begin{tabular}{|c|c|c|c|c|}
\hline \multirow{2}{*}{$\begin{array}{l}\text { Site of } \\
\text { Primary Tumour }\end{array}$} & \multicolumn{2}{|c|}{ KOH/PAS Positive } & \multicolumn{2}{|c|}{ KOH/PAS Negative } \\
\hline & $\begin{array}{l}\text { Primary } \\
\text { Tumour }\end{array}$ & Metastases & $\begin{array}{l}\text { Primary } \\
\text { Tumour }\end{array}$ & Metastases \\
\hline Colon & 35 & 55 & - & - \\
\hline Colon & - & - & 14 & 23 \\
\hline Caecum & 2 & 4 & - & - \\
\hline Rectum & 2 & 4 & - & - \\
\hline Stomach ${ }^{1}$ & 1 & 3 & - & - \\
\hline Stomach & - & - & 9 & 26 \\
\hline Small intestine & - & - & 1 & 2 \\
\hline Lung & - & - & 20 & 42 \\
\hline Breast & - & - & 3 & 4 \\
\hline Bile duct & - & - & 1 & 6 \\
\hline Ovary & 一 & - & 1 & 8 \\
\hline
\end{tabular}

Table Results of KOH/PAS staining on 89 mucinproducing adenocarcinomas and their 177 metastases, subdivided by the site of the primary tumour ${ }^{1}$ See text 
tumour showed the histological features of both colloid and adenocarcinoma.

In the table it will be seen that there was no $\mathbf{K O H} /$ PAS positive mucin (or other material) in either the primary tumours, which had arisen from tissues other than the gastrointestinal tract, or their metastases. This was felt to be significant since a biopsy of a solitary metastasis from several of these cases might have presented a problem to surgical pathologists asked to determine the site of the primary tumour since it would have been difficult to rule out the possibility of the lower gastrointestinal tract being the site.

It will be of interest that on two recent occasions we have been able to direct the attention of surgeons to the colon and rectum as primary sites based upon the presence of $\mathrm{KOH} / \mathrm{PAS}$ positive mucin in biopsies of the metastases.

\section{NORMAL TISSUES}

With the exception of a very pale red coloration seen in the liver, normal tissues from sites other than the lower gastrointestinal tract did not stain by the periodate-borohydride/ $\mathrm{KOH} / \mathrm{PAS}$ technique. A slight exception to this statement is the fact that myelin sheaths are positive but they present no difficulty in interpretation. Mucin in sections of terminal ileum (up to $30 \mathrm{~cm}$ from the caecum) may show a weak $\mathrm{KOH} / \mathrm{PAS}$ effect, and metastases from tumours arising in this area might be expected to give a similar result although such an assumption needs to be tested.

\section{Discussion}

The KOH/PAS effect has been shown by us both histochemically (Culling et al, 1974) and chemically (Reid et al, 1975) to be due to the presence of $\mathrm{O}$-acylated sialic acids in an epithelial mucin which is only produced by cells of, or cells derived from, the lower gastrointestinal tract of man. Further, we have demonstrated that this effect can be seen in isolation by first abolishing the normal PAS reactivity of tissue sections by oxidation of the existing vicinal diols (1:2 glycol groups) with periodic acid, followed by the reduction of the engendered aldehydes with sodium borohydride.

In this study it was found that only mucin gave a positive result by this technique and that, with one exception, such mucin had been produced either $(a)$ by normal epithelial cells in the caecum, colon or rectum (or the terminal ileum immediately adjacent to the caecum) or $(b)$ in the primary tumour, or in metastases from such a primary tumour which had arisen in this area.

The features of importance which arise from these observations are, in our opinion:

(1) Mucin-producing metastases from adeno- $\frac{\widehat{D}}{2}$ carcinoma of the lower gastrointestinal tract can be identified as such when the $\mathrm{KOH} / \mathrm{PAS}$ reaction is positive, such identification being of importance? when the site of the primary tumour is unknown. $\vec{\omega}$ On two occasions at the Vancouver General Hos- $\stackrel{\circ}{\circ}$ pital we have been able to provide surgeons with such infor mation.

(2) Mucin-producing primary adenocarcinoma $\underset{\infty}{\infty}$ of the lung (which will be $\mathrm{KOH} / \mathrm{PAS}$ negative) can of easily be distinguished from mucin-producing of metastases from adenocarcinoma of the lower 을 gastrointestinal tract when the primary tumour is $\rightarrow$ $\mathrm{KOH} / \mathrm{PAS}$ positive, which occurred in $70 \%$ of those we studied.

(3) The discovery that $30 \%$ of adenocarcinomas $\stackrel{\text { क }}{+}$ of the defined area of the lower gastrointestinal $\vec{\theta}$ tract are $\mathrm{KOH} / \mathrm{PAS}$ negative may prove to be of clinical significance. It may be surmised that this group of tumouis arises from a different cell type or that a greater degree of dysdifferentiation of the cells has taken place. A study is now being undertaken to determine the possible significance of this finding.

Supported by Medical Research Council of Canada, Grant \# 4375

\section{References}

Culling, C. F. A. (1974). Handbook of Histopathological and Histochemical Techniques, 3rd edition. Butterworths, London.

Culling, C. F. A., Reid, P. E., Clay, M. G., and Dunn, W. L. (1974). The histochemical demonstration of O-acylated $\mathrm{O}$ sialic acid in gastrointestinal mucins; their association $D$ with the potassium hydroxide-periodic acid-Schiff effect. J. Histochem. Cytochem., 22, 826-31.

Culling, C. F. A., Reid, P. E., and Dunn, W. L. (1971). The N effect of saponification upon certain histochemical reactions of the epithelial mucins of the gastrointestinal $\mathrm{N}$ tract. J. Histochem. Cytochem., 19, 654-662.

Reid, P. E., Culling, C. F. A., and Dunn, W. L. (1973). Saponification-induced increase in the periodic acid-Schiff reaction in the gastrointestinal tract: mechanism and 0 distribution of the reactive substance. J. Histochem. $\frac{\bar{\Phi}}{\Phi}$ Cytochem., 21, 473-482.

Reid, P. E., Culling, C. F. A., Tsang, W. C., Clay, M. G., Ramey, C. W., and Dunn, W. L. (1975). The demonstration of $\mathrm{O}$-acetylated sialic acids in colonic epithelial glycoproteins. Canad. J. Biochem., 53, 388-391. 